\title{
Estrategia del Océano Azul para el sector portuario (sincromodalidad y digitalización $)^{1}$
}

\section{Blue Ocean strategy for the port sector (synchromodality and digitization)}

\author{
N. Ortiz, N. González, B. Molina, F. Soler y A. Camarero \\ Recibido: marzo 24 de 2020 - Aceptado: junio 25 de 2020
}

\begin{abstract}
Resumen- La industria en general y los puertos en particular están derivando hacia procesos 4.0. El uso de nuevas tecnologías y pautas de mercado son las herramientas principales para el éxito de estos procesos. Los retos actuales del sector portuario pasan por desarrollar puertos Smart cuyas características principales se definen por la digitalización y la sincromodalidad. Solo ellas permiten determinar un nuevo escenario en el que es posible plantear la Estrategia del Océano Azul, dejando de lado la competencia y se buscando una nueva demanda. El creciente desarrollo de técnicas digitales y su implantación a los procesos portuarios de gestión y transporte, hace posible que esta estrategia sea viable en una dimensión aún mayor. La conclusión general de la investigación es que es que posible aplicar estrategias de mercado al sector portuario español con una metodología diferente a la empleada hasta el momento. Se observa que los puertos españoles aún tienen un largo camino que recorrer en materia de sostenibilidad, entendida en tres dimensiones: económica, social, y ambiental.
\end{abstract}

${ }^{1}$ Producto derivado del desarrollo de una tesina de Investigación "Estrategia del Océano Azul para el sector portuario (sincromodalidad y digitalización)"de la Universidad Politécnica de Madrid.

N. Ortiz, Universidad Politécnica de Madrid, Madrid, España, email: noemi.ortizrey@alumnos.upm.es

N. González, Universidad Politécnica de Madrid, Madrid, España, email: nicoleta.gcancelas@upm.es.

B. Molina, Universidad Politécnica de Madrid, Madrid, España,email: beatriz.molinas@alumnos.upm.es

F. Soler, Universidad Carlos III de Madrid, Madrid, España, email: fsoler@inf.uc3m.es

A. Camarero, Universidad Politécnica de Madrid, Madrid, España, email: alberto.camarero@upm.es

Como citar este artículo: Ortiz, N., González, N., Molina, B., Soler, F. y Camarero, A. Estrategia del Océano Azul para el sector portuario (sincromodalidad y digitalización), Entre Ciencia e Ingeniería, vol. 14, no. 27, pp. 50-57, enero-junio 2020. DOI: https://doi.org/10.31908/19098367.1768.

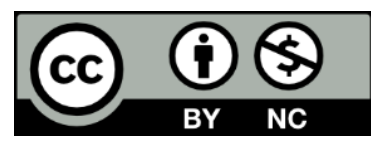

Attribution-NonCommercial 4.0 Intenational (CC By-NC 4.0)
Palabras clave- digitalización, Océano azul, puertos, sincromodalidad.

Abstract - Industry in general and ports in particular are moving towards 4.0 processes. The use of new technologies and new market patterns are the main tools for the success of these processes. The current challenges of the port sector are to develop Smart ports whose main characteristics are defined by digitalization and synchro-modality. Only they allow determining a new scenario in which it is possible to raise the Blue Ocean Strategy, leaving aside competition and looking for a new demand. The growing development of digital techniques and their implementation in port management and transport processes makes this strategy viable on an even greater scale. The general conclusion of the research is that it is possible to apply market strategies to the Spanish port sector with a different methodology to the one used until now. It can be seen that Spanish ports, still have a long way to go in terms of sustainability, understood in three dimensions: economic, social and environmental.

Keywords - digitalization, blue ocean, ports, synchromodality.

\section{INTRODUCCIÓN}

I OS puertos han jugado desde su existencia un papel fundamental en el comercio internacional. No obstante, su posición estratégica ha cambiado notablemente durante los últimos veinte años, en los cuales se ha venido produciendo una evolución hacia unos requisitos cada vez más exigentes de los estándares de operación y gestión.

Al mismo tiempo, el escenario global de competitividad portuaria ha sufrido cambios significativos. Todos los grandes puertos del mundo han sido testigos de una transición en el enfoque de su modelo de desarrollo económico, pasando de una apuesta por el crecimiento extensivo a una apuesta por el crecimiento intensivo [1]. El primero de ellos ponía el énfasis en aumentar la fuerza laboral o su capacitación, además de mejoras en las infraestructuras y mayores inversiones en capital y obtención de recursos. Por otro lado, el crecimiento intensivo se centra en adquirir una actitud innovadora, capaz de progresar hacia nuevas ideas y valores que impulsen 
mejoras en la eficiencia del sistema, aunque eso implique romper con los esquemas tradicionales. Este camino hacia el descubrimiento de nuevas y mejores metodologías permite perfeccionar la explotación de los recursos utilizables por una economía específica. Además, abre una nueva vía hacia desarrollos y mejoras sostenidos, e importantes saltos cualitativos en los niveles de bienestar [2].

Este cambio en la visión del funcionamiento portuario permite avanzar hacia modelos de gestión más transparentes y ampliar el foco de interés hacia otras áreas que favorezcan la competitividad portuaria más allá de las mejoras operacionales [3]. Para facilitar esta transformación, los puertos deben hacer uso de una forma efectiva de los recursos digitales y fortalecer su colaboración con la industria, fomentando de esta manera el valor añadido en las actividades portuarias [4].

Con todo ello, se puede decir que la transformación de los modelos de negocio y la innovación son dos imperativos para los puertos de todo el mundo a fin de aprovechar las oportunidades que brindan los mercados aún desconocidos [5].

Este proceso de mejora de la calidad de las operaciones portuarias por medio de la tecnología ha propiciado una progresiva liberalización de las actividades llevadas a cabo en los puertos del Sistema Portuario de Titularidad Estatal Español [6]. Con la intención de que estas actividades se produzcan respetando la libre competencia y evitando la formación de monopolios, se ha dotado a las Autoridades Portuarias de la capacidad para actuar sobre estas cuestiones. Además, estas tienen la responsabilidad de promover la innovación y la elaboración de estrategias encaminadas a mejorar la competitividad portuaria [7].

En definitiva, los servicios portuarios tradicionales pasan a ser competencia del sector privado, introduciendo así una mayor flexibilidad en la prestación de estos servicios. Como consecuencia, la transmisión de la información en el entorno portuario también sufre cambios. En las cadenas logísticas de los puertos confluyen tanto flujos físicos como de documentación, y los avances tecnológicos permiten agilizar los procesos de este flujo de información y mejorar de esta manera la oferta de servicios [8].

Finalmente, aparecen los conceptos de Smart Port (Puerto Inteligente) y Puerto 4.0 como consecuencia de este desarrollo tecnológico y las nuevas necesidades derivadas de su optimización. Las claves de este nuevo funcionamiento residen en la aplicación generalizada de la digitalización y la sincromodalidad a todos los procesos, sin olvidar otros aspectos esenciales como son la seguridad y la sostenibilidad. Se puede decir que la digitalización es el principal medio para la transformación 4.0 en los puertos [9].

La sincromodalidad, o intermodalidad sincronizada, se puede definir como el servicio que, mediante una planificación y gestión flexible y documentada, permite la toma de decisiones en cuanto a modo de transporte y ruta de cada elemento del sistema lo más tarde posible, dentro del proceso de planificación del transporte y del viaje mismo [10].

Cabe dejar bien clara su diferencia con la intermodalidad. Mientras que, en las soluciones de transporte intermodal, todas las modalidades se conocen previamente, en el transporte sincromodal se permite la flexibilidad para cambiar las modalidades en cualquier momento, logrando enviar la carga de una manera más eficiente y sostenible [11].
Es por ello necesario establecer un nuevo escenario rompedor para la sincromodalidad y la digitalización en el sector portuario que se pretende abordar en el presente estudio mediante la estrategia del Océano Azul.

\section{ESTADO DEL CONOCIMIENTO}

Los puertos marítimos han constituido a lo largo de la historia una fuente de riqueza para los territorios en los que se encuentran, ya sea a nivel municipal, regional, o nacional. Extendidos por todo el mundo, y dada su importancia para el desarrollo económico territorial, se ha evolucionado hacia una multitud de modelos organizativos y regulatorios [12].

Según apuntan numerosos estudios sobre la evolución del sector portuario, la globalización que está sufriendo actualmente el mercado mundial en todas sus facetas, con especial relevancia el crecimiento del comercio internacional de mercancías está propiciando un aumento de la competencia portuaria [13]. Este fenómeno es fácilmente apreciable en el proceso de jerarquización y selección que se está produciendo en los puertos españoles, donde se lucha por conseguir una mayor concentración de los tráficos portuarios y localización de empresas en su entorno más próximo, lo que conlleva además una aglutinación de los grupos tecnológicos en unas pocas áreas costeras [14].

En este contexto, se pueden distinguir dos corrientes. Una de ellas sería la formada por aquellos puertos que destacan por este desarrollo comercial, luchando por ofrecer una oferta de servicios mejor que la del resto, y postulándose por ser la mejor solución para las necesidades de los clientes. Estos puertos son unidades activas con capacidad para la toma de decisiones debido a su gran poder de influencia. Por el contrario, la segunda corriente sería la de aquellos puertos con un comportamiento más pasivo, alejado de la tendencia general de la globalización, y cuya importancia solo es percibida a nivel local.

Esta contraposición de comportamientos se manifiesta en las diferentes posturas que las Autoridades Portuarias del Sistema Portuario Español han adoptado en cuanto a cómo tiene que evolucionar el Marco Estratégico vigente. Para ilustrar el panorama actual, a principios del presente año se realizó un estudio a base de encuestas que las Autoridades Portuarias tenían que completar a fin de mostrar sus opiniones en cuanto a las diversas cuestiones que se les plantearon. Estas cuestiones versaban sobre temas como la gobernanza del sistema, la planificación y la gestión, o el régimen económicofinanciero.

\section{A. Estrategia océano azul}

La estrategia Océano Azul es una estrategia de mercado desarrollada por W. Chan Kim y Renée Mauborgne, profesores del INSEAD (una de las más prestigiosas escuelas de negocios del mundo), en la que se plantea que para llegar al éxito empresarial es necesario dejar de lado la competencia y buscar una nueva demanda, es decir, crear océanos azules.

El primero de los requisitos, referente a la competencia, significa que las compañías deben de dejar de competir entre ellas para alcanzar el éxito. Así, al conjunto de las empresas que existen actualmente en el mercado se las engloba dentro de los océanos rojos, mientras que las industrias aún 
desconocidas, aquellas que no existen en la actualidad, corresponden a los océanos azules. Los océanos rojos reciben este nombre porque la "competencia a muerte tiñe de sangre el agua del océano rojo".

Por otro lado, los océanos azules presentan una realidad alternativa conformada por los espacios no aprovechados del mercado y por la creación de una nueva demanda. En ellos no se compite, sino que se crean nuevas oportunidades de rentabilidad y crecimiento. Al no utilizar a la competencia como un elemento de referencia con el que compararse, estos océanos aplican una lógica estratégica diferente que se centra en la obtención de valor y en la innovación, la denominada innovación en valor. Esta tiene lugar cuando se consigue alinear la innovación con la utilidad, el precio y el coste, es decir, cuando se logran diferenciación y bajo coste al mismo tiempo. La diferenciación viene con la creación de elementos que la industria nunca ha ofrecido, con lo que se aumenta su valor para los compradores, mientras que la reducción de costes encuentra su máximo cuando se entra a operar en economías de escala (Fig. 1).

\section{Estrategia Océano Azul. \\ Principios: \\ - No competencia. \\ - Innovación en valor. \\ Herramientas: \\ - Matriz de las cuatro acciones. \\ - Curva de valor.}

Fig. 1. Innovación en valor [15].

Si se procede a la realización de un análisis histórico de la formación de industrias, se observa que un gran número de las que se conocen actualmente han surgido como océanos azules (automovilística, música, etc.), siendo desconocidas hasta el momento de su creación. De esta manera, se puede decir que los océanos azules siempre han existido, si bien es ahora cuando se los ha catalogado como tales. Así, la estrategia Océano Azul nace del estudio de estos nacimientos empresariales, analizando los rasgos que tienen en común y aquellos que los diferencian de las demás industrias, con la intención de encontrar un patrón común en ellos que permita la sistematización de su aplicación a nuevos casos (eliminando a su vez el riesgo). No obstante, a pesar de que lo aparentemente lógico sería considerar a la compañía o a la industria como las unidades de análisis, lo cierto es que es el movimiento estratégico el que mejor explica la creación de estos océanos, entendiendo como tal el conjunto de actuaciones y decisiones conducentes a la producción de una oferta importante que conlleve la creación de un mercado.

\section{B. Aplicación de la estrategia Océano Azul en los puertos}

Los puertos, cada vez más, son considerados empresas que prestan servicios muy diversos y que proporcionan un conjunto de infraestructuras. En consecuencia, como toda empresa, deben gestionarse con criterios empresariales. De acuerdo con esta premisa, los puertos españoles han adoptado paulatinamente una estrategia de negocio en su explotación.

Es por este comportamiento empresarial por el que resulta idónea la aplicación de la estrategia del Océano Azul para la elaboración de un nuevo modelo de gestión. La visión de negocio ha ido derivando en una competencia y rivalidad de cada vez mayores dimensiones entre los puertos del sistema y, a no ser que se tomen medidas al respecto, la creciente diferenciación entre los puertos que integran el sector puede debilitar su posición estratégica y potencial de generación de riqueza para el país.

Aplicar la estrategia del Océano Azul al sector portuario español sería algo novedoso, pues nunca se ha planteado en este mercado. Además, implicaría romper con toda la estrategia actual basada en un claro océano rojo de competencia, para lo cual sería necesario abandonar los prejuicios existentes acerca de cómo tiene que ser su funcionamiento, es decir, al ser un modelo que hasta ahora ha ido generando riqueza, podría parecer innecesario tratar de combatirlo. No obstante, es eso mismo lo que sugiere el océano azul, que se cuestione el funcionamiento actual de las cosas y se mire más allá de sus fronteras para abrir un nuevo mercado con un crecimiento y expectativas de futuro superiores a las de los mercados conocidos.

A la hora de emplear la estrategia en el modelo de negocios del sector portuario español, cabe destacar el hecho de que este presenta el extra de aunar sector público y sector privado, motivo que podría representar una dificultad añadida. Asimismo, es un sistema en el que convergen multitud de agentes, ya que los puertos son una parte más de la cadena logística del transporte de mercancías.

En esta línea se destaca la escasez de evidencias de aplicación de la estrategia Océano Azul al sector público (algunos gobiernos y autoridades regionales apuntan a su utilización para el desarrollo de nuevas políticas, pero no hay publicaciones concretas al respecto). Así, la aplicación de esta estrategia en el presente proyecto constituye una novedad no solo a nivel del sistema portuario estatal, sino a nivel del sector público español, e incluso mundial.

Por último, no se puede obviar el papel que la tecnología ejerce en esta propuesta. El creciente desarrollo de técnicas digitales y su implantación a los procesos portuarios de gestión y transporte, hace posible que esta estrategia sea viable en una dimensión aún mayor.

\section{III.METODOLOGÍA}

Se exponen a continuación los pasos a seguir para desarrollar la estrategia Océano Azul. Estos pasos están encaminados a caracterizar el mercado actual de la industria de estudio y a localizar posibles alternativas de desarrollo que la diferencien de los océanos rojos de dicho mercado. A la hora de caracterizar el mercado, es necesario realizar un estudio previo de este para conocer su situación actual y reconocer, a priori, sus fortalezas y debilidades. Posteriormente, se aplicará la secuencia de acciones propuesta por la estrategia Océano Azul. Con la información obtenida del estudio previo del mercado y las pautas marcadas por la estrategia, se obtendrán las posibles salidas de la industria en cuestión del mercado basado en la competencia. 


\section{A. Determinación del escenario de trabajo}

La determinación del escenario de trabajo se realizará mediante un diagnóstico y análisis del estado del arte. Para ello, se revisará su situación actual y se identificarán los aspectos que aportan información esencial para el desarrollo de la estrategia.

\section{B. Estrategia Océano azul}

La estrategia por desarrollar consiste en seguir los pasos que se presentan en Kim \& Mauborgne [16] y que se resumen a continuación (Fig. 2).

Reconstruir la frontera del marcado
•-Explorar sectores alternativos: ofrecer algo nuevo
•-Explorar la cadena de compradores: desafiar el objetivo
•-Explorar productos y servicios complementarios
•-Explorara el atractivo funcional y emocional

Fig. 2. Pasos metodología Kim \& Mauborque [16].

\section{Reconstrucción de las fronteras del mercado}

El principio de la estrategia Océano Azul que se debe aplicar en primer lugar es la reconstrucción de las fronteras de mercado. Para ello, de entre todas las posibilidades que ofrece el mercado, hay que identificar cuáles de todas ellas constituyen oportunidades comercialmente atractivas para la creación de estos océanos.

En el libro de Kim \& Mauborgne [15], se describen seis enfoques fundamentales para reconstruir estas fronteras del mercado, los cuales surgen a partir de la identificación de patrones sistemáticos empleados en la creación de océanos azules. Estos patrones, durante su estudio, fueron agrupados bajo el esquema de las seis vías, las cuales se sustentan en la capacidad de ver las realidades conocidas con una nueva perspectiva. Por lo general, son aplicables a cualquier sector de la industria.

\section{Enfocarse en la perspectiva global y no en las cifras}

Este segundo principio persigue la alineación del proceso de planeación estratégica con el objetivo de centrar la atención en la perspectiva global y aplicar las ideas para dibujar el cuadro estratégico de la industria de tal forma que represente un océano azul. Se trata de un enfoque a partir del cual se reduce el riesgo de la planeación alrededor de una serie de movimientos en el interior del océano rojo.
La utilización de un cuadro estratégico, como este principio propone, ayuda a centrar la atención de la estrategia en la perspectiva global en lugar de en las cifras y la terminología, impidiendo por tanto quedar atrapado en los detalles operativos. Se puede decir que funciona como una alerta para reconocer la necesidad de cambiar, ayudando a predecir y plantear el crecimiento y las utilidades futuras de la industria.

\section{E. Ir más allá de la demanda existente}

El propósito de este tercer principio es maximizar el tamaño del océano azul que se está creando. Ir más allá de la demanda existente implica agregar la mayor demanda posible al nuevo producto o servicio, atenuando así el riesgo de escala asociado con la creación de un nuevo mercado.

Para lograr el éxito de este enfoque, se tiene que dejar de lado la estrategia común de los océanos rojos de mantener a sus clientes existentes y aumentar su volumen de negocios con ellos. Esta fijación en los clientes existentes, y su correspondiente segmentación en grupos cada vez más finos para satisfacer mejor las preferencias de estos, induce a una competencia intensa y a la creación de mercados objetivo excesivamente reducidos.

Sin embargo, para crear océanos azules hay que seguir el camino contrario. La industria se debe fijar en los no clientes y debe aprovechar las cosas que valoran en común todos los compradores, consolidando de esta manera los segmentos hasta ahora diferenciados. Así es como se consiguen clientes nuevos que antes no formaban parte del mercado. El reto de este principio es intuir con acierto quiénes son los no clientes de la industria y cómo exprimir su potencial.

Finalmente, se debe aprovechar el tamaño del nuevo océano azul para que el resultado sea sostenible y beneficioso para todos. Esta última cuestión se explicará en el siguiente apartado.

\section{F. Aplicar la secuencia estratégica correcta}

El cuarto principio de la estrategia Océano Azul se fundamenta en la construcción de un modelo de negocios fuerte, mediante la ordenación y validación de las ideas para océanos azules a fin de asegurar su viabilidad comercial. La claridad en la secuencia en que se deben suceder las actuaciones y su validación reduce drásticamente los riesgos del nuevo modelo de negocios.

\section{G.Análisis de resultados y conclusiones}

Una vez desarrollada la estrategia del océano azul aplicado al sistema portuario será el momento de analizar los resultados y obtener conclusiones

\section{RESULTADOS DE LA APLICACIÓN DE LA ESTRATEGIA OCÉANO AZUL}

\section{A. Resultados de la determinación del escenario de trabajo}

Para determinar el escenario de trabajo se recopilaron las principales herramientas tecnológicas de la revolución 4.0 y su posible aplicación a los puertos marítimos y demás elementos de la cadena logística. No obstante, fue un estudio general en el que se aportaron datos y ejemplos globales, por lo que ahora se aportarán datos relativos al sistema español. Los diferentes aspectos sobre los que se hará la comparativa tecnológica de 
los puertos españoles son: logística, movilidad, medio ambiente, economía, factor social, y gobernanza.

\section{B. Estrategia Océano Azul}

Para la investigación desarrollada se optó ir por la quinta vía de la estrategia del Océano Azul que se presenta a continuación.

\section{1) Explorar el atractivo funcional o emocional para los compradores}

La forma de competir que tienen las industrias puede ser de dos tipos: atendiendo a su atractivo racional, para lo que se basan en su precio y función, o atendiendo a su atractivo emocional, cuando compiten principalmente basándose en los sentimientos. Sin embargo, el atractivo final de los productos y servicios ofrecidos por las industrias proviene de las dos partes. Esto es así porque el comportamiento de las compañías a lo largo del tiempo crea, inconscientemente, expectativas en los consumidores.

En estos casos, para salir de los océanos rojos y descubrir espacios desconocidos, es necesario que las compañías se cuestionen su orientación funcional o emocional. Para ello, pueden recurrir a las siguientes preguntas: ¿compite su industria sobre la base del atractivo funcional o emocional? Si su industria compite con base en el atractivo emocional, ¿cuáles son los elementos qué podrá eliminar a fin de darle un giro funcional? Si compite con base en la funcionalidad, ¿cuáles son los elementos que podrá agregar para darle un giro emocional?

Dando respuesta a las preguntas, la primera de ellas es clara: el sector portuario español compite en base a su atractivo funcional. Este atractivo, como ya se ha discutido anteriormente, se refuerza mediante inversiones en los grandes puertos comerciales (como el Puerto de Barcelona), los cuales son los que pueden exportar al exterior una imagen de un sistema potente y eficiente, $\mathrm{y}$, como consecuencia, los puertos de menor entidad pierden relevancia en el sistema portuario.

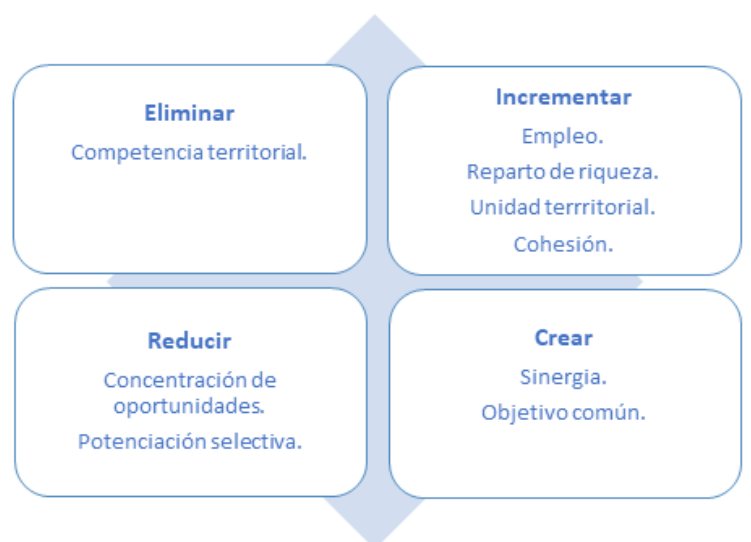

Fig. 3. Matriz de las cuatro acciones. Quinta vía: explorar el atractivo funcional o emocional para los compradores.

Ante el descontento patente que existe en los puertos más pequeños, para dar al sistema un giro emocional, se debe fomentar el concepto de unidad, es decir, fomentar una participación clara, real, y conjunta de todos sus puertos integrantes (lo que se traduce en evitar concentrar la oferta en unos determinados puntos). Este giro, además, traería consigo un crecimiento económico más uniforme en el territorio nacional, un reparto más justo de la riqueza, y un fomento del empleo.

Las variables que se utilizarán para la definición del mercado actual atrapado en un océano rojo y el futuro océano azul, y que, por tanto, tienen como misión efectuar el giro emocional al caso de estudio, son las que se proponen a continuación (Fig. 3)

Su curva de valor correspondiente es la que se representa en la Fig. 4.

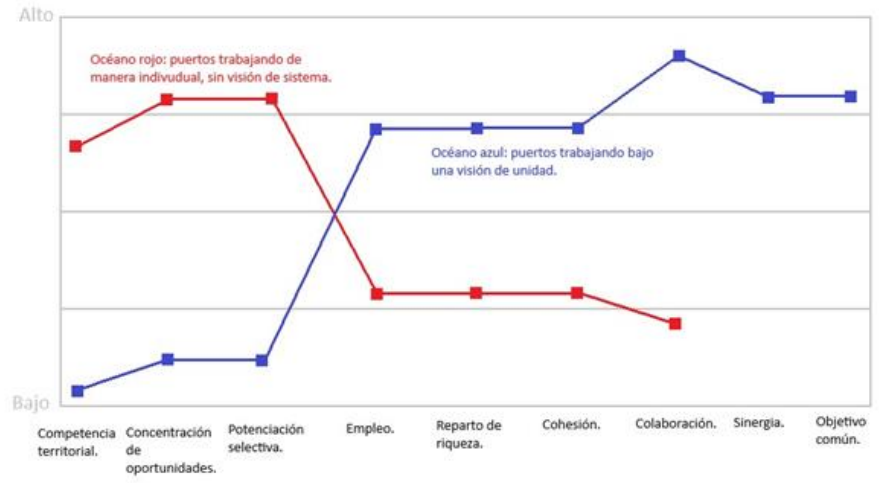

Fig. 4. Curva de valor. Quinta vía: explorar el atractivo funcional o emocional para los compradores.

\section{2) Océano azul}

Partiendo una vez más de la identificación de colonos, y teniendo en cuenta que para asegurar una correcta transición entre los dos escenarios estos no pueden desaparecer completamente, pero sí ver mermado su valor, se pueden encontrar los aquí expuestos:

Las inversiones (públicas y privadas). La razón de mantener las inversiones es la clara necesidad que la industria tiene de ellas. En el nuevo océano, por ejemplo, es necesario invertir en infraestructura ferroviaria (la oferta actual no hace frente a la demanda). Sí cabe destacar que estas inversiones ya no podrían verse como un instrumento para fomentar la competencia, sino como una herramienta de homogenización de oportunidades.

Finalmente, los pioneros que más clara diferencia suponen entre los dos escenarios, ofreciendo un valor sin precedentes, y constituyendo las fuentes más poderosas de crecimiento son:

- Tasas dinámicas

- Cadena logística digitalizada y sincromodal

- Visión de conjunto

En el caso de las tasas dinámicas, la innovación vendría de manos de la digitalización y nuevas tecnologías de soporte del concepto, mientras que el valor vendría de manos del proceso de elección de los precios, imparcial y transparente. En lugar de que cada Autoridad Portuaria establezca los precios que mejor le convengan para asegurar su autosuficiencia y atraer más tráfico que sus competidores, un sistema centralizado, digital, y automático, basado en la toma de decisiones en tiempo real, sería el encargado de desempeñar esta función de acuerdo con la información aportada por cada puerto en cada momento.

La cadena logística digitalizada y sincromodal aporta innovación de nuevo por medio de la tecnología. La conexión entre modos y la elección de ruta y modo en tiempo real, 
además del aporte de información sobre el estado de las infraestructuras, supone un ahorro energético y de tiempo, y una optimización de los procesos. El valor vendría dado por esta optimización y por la prestación de un servicio, tanto marítimo como terrestre, más flexible (su capacidad de adaptación a los condicionantes del entorno de una forma instantánea). Dentro de esta cadena logística de transporte ya estaría plenamente integrado el ferrocarril.

En cuanto a la visión de conjunto, esta hace referencia a un nuevo modelo de gestión. Este cambio de modelo implica innovación, a la vez que supone un salto en valor gracias al fomento de la colaboración entre puertos, la transparencia de los procesos y la toma de decisiones, y el intercambio de información.

El resultado final de este mapa de pioneros, emigrantes, y colonos es la que se muestra en la Fig. 5.

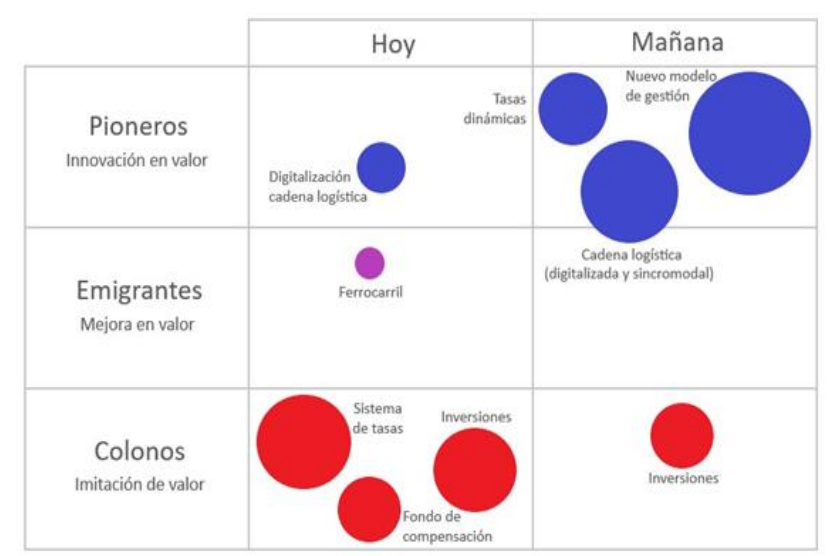

Fig. 5. Mapa PEC. Perspectiva global del océano azul para el sector portuario español.

\section{3) Resultados de ir más allá de la demanda existente}

El objetivo de este tercer principio de la estrategia es estudiar la forma de maximizar el tamaño del océano azul creado. Para ello, hay que buscar la demanda más allá de las fronteras establecidas por el mercado actual.

La forma de traspasar las fronteras es mediante la identificación de los no clientes de la industria, los cuales son clasificados en tres niveles. Se procede a continuación a la labor de identificación.

Empezando por los no clientes de primer nivel, se puede recurrir a la siguiente pregunta para ayudar a su definición: ¿cuáles son las razones principales por las cuales los no clientes del primer nivel están dispuestos a saltar del barco y abandonar su industria? Esta pregunta ha de ser enfocada desde una perspectiva unificadora, la cual sirva para consolidar a su vez el mercado de los compradores, ampliándolo.

Su aplicación al caso del sector portuario español podría ser mediante la identificación, en primer lugar, de los clientes, representados por aquellos tráficos de buques cautivos de un selecto número de puertos por ser los únicos capaces de ofrecer un servicio adecuado a sus necesidades. Este podría ser el caso de los grandes puertos especializados en la contenerización. Por otro lado, los no clientes de primer nivel, aquellos compradores que utilizan mínimamente el sistema, y piensan abandonarlo en cuanto encuentren algo que consideren mejor, serían aquellos buques que operan en los puertos de menor tamaño (que en consecuencia presentan un índice de especialización menor), pero que tan pronto pudieran hacerlo en otro tipo de puertos, se marcharían a estos.

Siguiendo con los no clientes de segundo nivel, estos no clientes no tienen intención de formar parte del mercado porque consideran que está fuera de su alcance o porque no cumple sus expectativas. Un ejemplo de este nivel serían aquellos tráficos que prefieren operar en puertos de otros países cercanos (como sería el norte de África) porque a su juicio los precios de los puertos de estos países son más competitivos.

En último lugar, los no clientes de tercer nivel son aquellos clientes potenciales que nunca han sido considerados por la industria, los inexplorados. En este sentido, se pueden identificar en este papel a los propios puertos del sistema, más concretamente, a los puertos más pequeños. Para llegar a esta conclusión, se ha asumido que estos puertos deben empezar a verse como receptores de productos y servicios, es decir, como receptores de oportunidades para conseguir equilibrar el sistema.

Echando la vista atrás hacia las posibilidades de creación de océanos azules en el sistema portuario, se obtiene la respuesta a la unificación de los tres niveles de no clientes, a la vez que a la consolidación de los que ya forman parte del mercado. Esta respuesta a la ampliación de la demanda está en ofrecer un servicio capaz de satisfacer al mismo tiempo a los cuatro niveles en cuestión. La solución planteada en este caso es la creación de alianzas entre los puertos del sistema, es decir, su funcionamiento como un conjunto, y el sistema de tasas dinámicas.

Para los no clientes de primer nivel, el aspecto fundamental que les hace susceptibles de marcharse del mercado es la falta de especialización de los puertos de menor entidad del sistema. Con una política de inversiones más justa que evite situaciones de sobrecapacidad de infraestructuras portuarias en los puertos que más invierten en crecimiento, y desvíe las inversiones hacia aquellos puertos en una clara situación de desventaja competitiva, se lograría evitar la marcha de estos no clientes de primer nivel. Al mismo tiempo, aumentaría la confianza en el sector. Por otro lado, los ya clientes del sector tampoco perderían calidad en el servicio que reciben (ya existe una sobrecapacidad de infraestructuras marítimas; no invertir en ellas en los grandes puertos no supone una pérdida de capacidad).

Los no clientes de segundo nivel también se beneficiarían de esta medida. El sistema de tasas dinámicas favorecería la oferta de precios más ajustados a las necesidades de cada momento y podría suponer una solución instantánea a la captación de aquellos tráficos que operan en puertos de países cercanos. Además, la adopción de los valores de los precios se ejecutaría a través de software, capaz de tomar decisiones de forma instantánea de acuerdo a los resultados de los análisis de la información a él proporcionada. Por este motivo, serían decisiones encaminadas a lograr el equilibrio del sistema, y tomadas de una manera transparente e imparcial.

Finalmente, en lo que respecta a los no clientes de tercer nivel, la mejora de su posición estratégica en el sector portuario nacional $\mathrm{y}$, por consiguiente, en el panorama internacional, se llevaría a cabo gracias a las dos medidas planteadas. Ambas soluciones representan para los puertos de 
menor entidad una oportunidad para aumentar sus tráficos y fomentar la economía local y cohesión territorial.

4) Resultados de aplicar la secuencia estratégica correcta

El fin de este último principio de la estrategia es garantizar la viabilidad comercial del modelo propuesto. Para alcanzar el objetivo, hay que seguir los pasos indicados en la metodología, los cuales consisten en asegurar que el océano azul funciona en las áreas de utilidad para el comprador, precio, coste, y adopción.

Considerando la utilidad para los clientes, el primer paso es analizar a través de las palancas propuestas por los creadores de la estrategia, cómo el sector portuario puede activarse para ofrecer una utilidad excepcional a sus clientes. Estas palancas son la productividad para el cliente (referida a la ayuda que brinda el producto o servicio al consumidor para hacer las cosas mejor o más rápidamente), la simplicidad, la comodidad, el riesgo, la imagen, y el medio ambiente, y se aplican al ciclo de experiencia del comprador. Para el análisis, se procede a la elaboración de la tabla que se muestra en la Tabla I.

TABLA I.

RESULTADO DE APLICAR LA SECUENCIA ESTRATÉGICA CORRECTA

\begin{tabular}{|c|c|c|}
\hline & Compra & Uso \\
\hline Productividad & $\mathrm{X}$ & $\mathrm{X}$ \\
\hline Simplicidad & $\mathrm{X}$ & $\mathrm{X}$ \\
\hline Comodidad & & $\mathrm{X}$ \\
\hline Riesgo & $\mathrm{X}$ & \\
\hline Imagen & $\mathrm{X}$ & \\
\hline Medio ambiente & $\mathrm{X}$ & $\mathrm{X}$ \\
\hline
\end{tabular}

Las aspas representan aquellas fases que gracias al océano azul experimentan un aumento de su utilidad en cada área propuesta por las seis palancas. En general, se puede apreciar como el nuevo mercado ofrece mejoras en todas las áreas que los compradores, tanto objetiva como subjetivamente, analizan a la hora de elegir un servicio.

La productividad, por su parte, mejora en los dos niveles del ciclo de experiencia del comprador. En la primera fase de compra, los buques no tendrían que decidir en qué puerto operar o establecer relaciones comerciales, reduciendo así esfuerzos, sino que el sistema portuario se encargaría de esa toma de decisiones en tiempo real de una forma completamente digital, transmitiendo esa decisión al buque. También podría verse como la ayuda del concepto de sincromodalidad a la toma de decisiones en cuanto a modo de transporte y ruta dentro de la cadena logística, las cuales se harían de una forma más eficiente. Posteriormente, en la fase de uso, se ve como esta categoría se ve optimizada gracias a la reducción de los tiempos de espera. El sistema dinámico de reparto de buques entre los diferentes puertos que conforman el sector portuario español, al basar su funcionamiento en evitar colapsos y tiempos de espera, repercute de manera positiva en la operación de los buques en el sistema portuario.

En cuanto a la simplicidad, tanto la estandarización de trámites, como la digitalización homogénea de los puertos, y el sistema de tasas dinámicas, contribuyen a minimizar la complejidad existente en el sistema actual. Las fases de compra y uso volverían a ser las grandes beneficiadas.

La comodidad, por otra parte, entendida como la posibilidad que tienen los compradores de satisfacer sus necesidades las veinticuatro horas del día, los 365 días del año, tendría su gran mejora en la fase de uso. La aplicación de tecnología permitiría reducir la dependencia sobre trabajadores, cuya disponibilidad y rendimientos son variables a lo largo del día.

En lo referente al riesgo, su reducción se puede efectuar en el plano económico, físico, o emocional, incluyendo la reputación. Así, el océano azul aporta una utilidad excepcional con respecto al océano rojo gracias a, sobre todo, la transparencia. Este hecho se manifiesta en la fase de compra.

La imagen, relacionada con la actitud del sistema portuario a la hora de ofrecer sus servicios, también logra su mayor utilidad en la fase de compra. La flexibilidad del sector portuario para hacer frente a demandas variables y su capacidad de adaptación a las circunstancias, proporciona a los buques una imagen de sistema sólido a la vez que dinámico, capaz de adelantarse a los problemas y evitarlos antes de que sucedan.

Finalmente, los compradores sentirían que su compromiso con el medio ambiente se ve satisfecho gracias a los diversos sistemas de optimización de las operaciones en los puertos, que estarían totalmente digitalizadas y monitorizadas. De esta forma, los tiempos y consumos de combustible serían mucho menores, repercutiendo en una mayor utilidad en ambas fases.

\section{V.CONCLUSIONES}

La conclusión general a la que se ha llegado con la realización de esta investigación es que es que posible aplicar estrategias de mercado al sector portuario español con una metodología diferente a la empleada hasta el momento. Esto significa que para poder hacer frente a los retos futuros del sistema de cara a la globalización y evitar su estancamiento, el sistema portuario estatal debe mirar hacia otras alternativas estratégicas que le ayuden a vislumbrar oportunidades. Así, la estrategia Océano Azul, considerada idónea para este cometido por sus excelentes resultados en otros sectores de la economía, ha puesto sobre la mesa una serie de cuestiones que los puertos españoles deben abordar para crear un nuevo mercado que no se base en la competencia.

\section{A. Conclusiones de la reconstrucción de las fronteras de mercado}

A raíz de los resultados obtenidos de las seis vías, se observa que los puertos españoles aún tienen un largo camino que recorrer en materia de sostenibilidad, entendida en sus tres dimensiones: económica, social, y ambiental.

En la dimensión económica, se extrae que la variable de colaboración entre puertos es la que mayor valor tiene. Esto es así porque solo con colaboración es posible que haya un flujo veraz y transparente de información que permita el establecimiento de un objetivo común que satisfaga las motivaciones del conjunto de los puertos del sistema español.

De la dimensión social, por su parte, se concluye que la variable con un valor más alto es la cohesión territorial. El mercado actual de competencia acentúa las diferencias territoriales, y seguirá haciéndolo si no cambia sus reglas. Por este motivo, se hace casi obligatorio la puesta en marcha de iniciativas que transformen el pensamiento racional del sector y lo lleven a un plano más emocional. La Estrategia Océano 
Azul ha cumplido en este sentido con su propósito.

Por último, de la dimensión ambiental se puede decir que la variable cuyo valor es más elevado es la sincromodalidad. Solo mediante su funcionamiento gracias a la implantación del ferrocarril para el transporte de mercancías desde y hacia los puertos, y de los procesos digitales, se puede reducir la dependencia de la carretera. De nuevo, el océano azul ha conseguido poner de manifiesto los puntos débiles del sector portuario hasta ahora conocido y proponer soluciones innovadoras con un alto valor para el conjunto de los agentes implicados.

\section{B. Conclusiones de enfocarse en la perspectiva global y no en las cifras \\ La aplicación del segundo principio de la estrategia ha} concluido en que la mayor parte de las medidas se han propuesto para el impulso de un nuevo océano son medidas de innovación en valor, evitando así caer en el error hasta ahora cometido de la imitación de valor. Su representación gráfica permite, además, ilustrar de una manera sencilla este fenómeno.

\section{Conclusiones de ir más allá de la demanda existente}

La búsqueda de los no clientes de la industria que propone el tercer principio de la estrategia ha concluido en una ampliación de los agentes identificados hasta este momento como la demanda del mercado. Así, el océano azul cumple su promesa de aumento de la demanda.

\section{Conclusiones de aplicar la secuencia estratégica correcta}

Este cuarto principio del océano azul ha permitido vislumbrar aquellos aspectos que pueden suponer una barrera para el desarrollo de las medidas propuestas, y sus soluciones correspondientes.

\section{REFERENCIAS}

[1] Panayides, P. M. "Global supply chain integration and competitiveness of port terminals. In Ports, cities, and global supply chains". Routledge,2017, pp. 43-56.

[2] Zereik, E., Bibuli, M., Mišković, N., Ridao, P., \& Pascoal, A. (2018) Challenges and future trends in marine robotics. Annual Reviews in Control, 46, pp. 350-368.

[3] Heilig, L., Lalla-Ruiz, E., \& Voß, S. (2017). Digital transformation in maritime ports: analysis and a game theoretic framework. Netnomics: Economic research and electronic networking, 18(2-3), pp. 227-254.

[4] Heilig, L., Schwarze, S., \& Voß, S. "An analysis of digital transformation in the history and future of modern ports". 2017.

[5] Acciaro, M., Renken, K., \& El Khadiri, N. "Technological Change and Logistics Development in European Ports". In European Port Cities in Transition (pp. 73-88). Springer, Cham. 2020.

[6] Dooms, M., Van Der Lugt, L., Parola, F., Satta, G., \& Song, D. W. (2019). The internationalization of port managing bodies in concept and practice. Maritime Policy \& Management, 46(5), pp. 585-612.

[7] Tavasszy, L., Behdani, B., \& Konings, R. "Intermodality and synchromodality. In Ports and Networks". Routledge, 2017, pp. 251266.

[8] Castelein, B., Geerlings, H., \& van Duin, R. (2020). The reefer container market and academic research: a review study. Journal of Cleaner Production, 120654. 2020.

[9] Douaioui, K., Fri, M., \& Mabrouki, C. "Smart port: Design and perspectives". In 2018 4th International Conference on Logistics Operations Management (GOL) (pp. 1-6). IEEE.

[10] Dong, C., Boute, R., McKinnon, A., \& Verelst, M. (2018). Investigating synchromodality from a supply chain perspective.
Transportation Research Part D: Transport and Environment, 61, 4257.

[11] Zhang, M., \& Pel, A. J. (2016). Synchromodal hinterland freight transport: Model study for the port of Rotterdam. Journal of Transport Geography, 52, 1-10.

[12] Dooms, M., Van Der Lugt, L., Parola, F., Satta, G., \& Song, D. W. (2019). The internationalization of port managing bodies in concept and practice. Maritime Policy \& Management, 46(5), 585-612.

[13] Zhang, X., \& Roe, M. "Port Competition. In Maritime Container Port Security". Palgrave Macmillan, Cham, 2019, pp. 59-85.

[14] Garcia-Alonso, L., Monios, J., \& Vallejo-Pinto, J. Á. (2019). Port competition through hinterland accessibility: the case of Spain. Maritime Economics \& Logistics, 21(2), pp. 258-277.

[15] Mauborgne, R., \& Kim, W. C. (2005). La estrategia del océano azul. Harvard Deusto business review, (131), 22-31.

[16] Kim, W. C., \& Mauborgne, R. (2004). La estrategia del océano azul. Harvard Business Review, 82(10), 2004.

Noemí Ortiz Rey. Ingeniera de Caminos, Canales y Puertos por la Universidad Politécnica de Madrid y Master Ingeniero de Caminos, Canales y Puertos por la Universidad Politécnica de Madrid en la especialidad en Servicios y Transportes Urbanos.

ORCID: https://orcid.org/ 0000-0001-7923-3584

Nicoleta González-Cancelas. Doctora Ingeniera de Caminos, Canales y Puertos por la Universidad Politécnica de Madrid. Es especialista en logística y transporte. Actualmente desarrolla su actividad como Profesora Contratada Doctora del Departamento de Ingeniería Civil, Transporte y Territorio de la Escuela Técnica Superior de Ingenieros de Caminos, Canales y Puertos de la Universidad Politécnica de Madrid al tiempo que es profesora del Máster Universitario en Sistemas de Ingeniería Civil (MUSIC) de la Escuela Técnica Superior de Ingenieros de Caminos, Canales y Puertos de la Universidad Politécnica de Madrid y del Master en Gestión y Planificación Portuaria e Intermodalidad; Master del Campus Portuario de Puertos del Estado. Es especialista en Logística y Transporte.

ORCID: https://orcid.org/ 0000-0001-7167-1563

Beatriz Molina Serrano. Doctora Ingeniera de Caminos, Canales y Puertos por la Universidad Politécnica de Madrid. Su experiencia está relacionada principalmente con la seguridad vial y la gestión de infraestructuras viarias, si bien relacionada con la gestión marítima portuaria. Actualmente desarrolla su actividad como jefe de proyectos en el sector privado. ORCID: https://orcid.org/0000-0002-7832-9573

Francisco Soler-Flores. Licenciado en Matemáticas por la Universidad de Almería. Doctor en Tecnología y Sistemas de Información por la Universidad de Castilla-La Mancha. Profesor de la Universidad Carlos III de Madrid (Departamento de Informática). Es especialista en Analytics y Machine Learning. ORCID: https://orcid.org/ 0000-0002-1636-834X.

Alberto Camarero-Orive. Doctor Ingeniero de Caminos, Canales y Puertos por la Universidad Politécnica de Madrid y Licenciado en Economía y en Administración, y Dirección de Empresas por la Facultad de Ciencias Sociales y Jurídicas de la Universidad Carlos III de Madrid. Profesor titular del Departamento de Ingeniería Civil y Transportes en la Unidad Docente de Explotación de Puertos. Además, es Profesor del Programa de Doctorado "Ingeniería e Infraestructura de los transportes".

ORCID: https://orcid.org/ 0000-0002-0614-8714. 